\title{
Screening of Lectins for Specific Detection of Listeria Monocytogenes
}

\begin{abstract}
In the present study, a specific binding activity between lectin and Listeria monocytogenes was studied by agglutination assay. The ten lectins were screened for their agglutination activity (HA) against $L$. monocytogenes ATCC 15313, 19111, 19115, 19118, 13932 \& BAA 751 along with lab isolates of RM -17 and RM 26. Wherein $84.4 \pm 1.76 \%$ binding was observed with wheat germ agglutinin (WGA) this was followed by $70 \%$ with Helix pometia agglutinin(HPA), $51.25 \%$ with Arachis hypogea lectin (AHL), 39.375\% with Ulex europeas lectin (UEL), 30.625\% with Lens culinaris lectin (LCL), and $18.75 \%$ with Sambucus nigra agglutinin (SNA), $26.25 \%$ with Phytolaccka Americana(PAL), 31.25\% Maackia amurensis lectin (MAL) and 16.25\% concavalin A. But, $<10 \% \mathrm{HA}$ was observed with Pisium Sativum (PSA). Additionally, these lectins were also screened for binding activity with Gram positive and Gram negative bacteria other than $L$. monocytogenes, but, no agglutination was observed except Salmonella (10-20\%), Klebsiella (10-20\%), and Citrobacter (45\%). The HA of WGA with $L$. monocytogenes was observed under scanning electron microscopy having cell aggregation with gapped and patchier cells, but, in some cases cell rupture was also seen. It was clear from the sugar inhibition assay that the HA of WGA with L. monocytogenes was reliably inhibited in presence of $\mathrm{N}$-acetylglucosamine (NAGA) at a rate of $0,35,50,80$ and $100 \%$, respectively, at $0,0.1,0.25,0.5,0.75$ and $1.0 \mathrm{M}$ concentration, respectively. Whereas in the case of glucose, the $\%$ inhibition was less compared to WGA followed by galactose, in the case of mannose, a least inhibition of $25 \%$ was observed at $1 \mathrm{M}$ concentration. The complete inhibition of agglutination in the presence of NAGA indicates that the binding of L. monocytogenes cells to WGA lectin was NAGA specific. These results will help in the use of WGA in the development of rapid test for detection of L. monocytogenes in milk.
\end{abstract}

Keywords: Lectin; Listeria monocytogenes; Agglutination
Research Article

Volume 5 Issue 3 - 2017

Raghu HV*, Naresh Kumar, Mayuri, Komal Arya and Pradeep Kumar Sharma

National Dairy Research Institute, Deemed University, India

*Corresponding author: Raghu HV, Dairy Microbiology Division and National Referral centre, ICAR-National Dairy Research Institute, Deemed University, Karnal-132001, India, Tel: +91-184-2259534, +91-9466963599;

Email: raghu@ndri.res.in, 4rvsy.dmndri@gmail.com

Received: May 09, 2017 | Published: July 19, 2017
Abbreviations: WGA: Wheat Germ Agglutinin; PSA: Pisium Sativum; HPA: Helix Pometia Agglutinin; SNA: Sambucus Nigra Agglutinin; PAL: Phytolaccka Americana; LCL: Lens culinaris Lectin; UEL: Ulex Europeas Lectin; MAL: Maackia Amurensis Lectin; FSSAI: Food Safety Standard Authority of India; ATCC: American Type Culture Collection; EMCCD: Electron Multiplying Charged Coupled Device

\section{Introduction}

Listeria monocytogenes is the causative agent of listeriosis, a disease that can be serious and is often fatal in high risk groups such as pregnant women, neonates, and immunocompromised adults with a rate which may reach $75 \%$ [1]. Different food types including raw vegetables, raw milk, soft cheeses, fish, poultry, processed chicken, and beef have been found to be contaminated with L. monocytogenes [2]. In particular, the ability of the organism to grow at refrigeration temperatures [3] and on dry surfaces [4] and its ability to tolerate acidic conditions make it well adapted to food environments which normally restrict bacterial growth. The primary sources of L. monocytogenes in milk and dairy products include the feed, bedding, vegetation, soil, animal faeces, contaminated water, diseased and unclean udders and teats, human hands and handling equipment [5].
Both facts, i.e. occurrence of L. monocytogenes in food [6] and the risk of contracting listeriosis have been very well established. The linkage of dairy products to both invasive and noninvasive listeriosis outbreaks is well known [7]. Various outbreaks have been associated with raw milk, pasteurized milk [8], soft cheeses [9], Crave Brothers Farmstead cheeses [10], hard cheese [11], semisoft cheese [12] and ice cream [13] (CDC, 2015). The food safety regulations of some countries (e.g. USA) require zero tolerance of L. monocytogenes in ready-to-eat (RTE) foods [14]. For others, therefore, L. monocytogenes has also become an issue of global concern because of its increased presence in milk and other food products [15]. Recently, Food Safety standard Authority of India (FSSAI) has established a microbiological criteria for dairy products that L. monocytogenes should be absent in per $25 \mathrm{~g}$ of every dairy product in India.

Conventional methods for the detection of food pathogens, although typically sensitive, often require multiple timeconsuming steps such as extraction, isolation, enrichment, counting, etc., prior to measurement, resulting in testing times which can be in days [16]. Rapid detection of foodborne pathogens is a key step in the control of food related diseases. There is an urgent necessity to develop rapid and sensitive detection methods. The advent of new technologies, namely biosensors, has brought 
in new and promising approaches comparable to or better than the conventional analytical systems in terms of performance (e.g. reliability, sensitivity, selectivity, specificity and robustness), that can identify the contaminants much faster, more efficiently and can give effective real-time monitoring.

A range of molecules with bio-recognition powers are available naturally such as antibodies, enzymes, and nucleic acids, which are used in the development of rapid techniques for detection. Lectins are carbohydrate-binding proteins other than enzymes or antibodies that exist in most living organisms, ranging from viruses and bacteria to plants and animals [17]. In particular, their role in cell recognition, as well as their employment as invaluable tools for the study of complex carbohydrates in solution and on cell surfaces, is contributing markedly to advances in glycobiology. Lectins interact with carbohydrates, non-covalently in a manner that is usually reversible and highly specific [18]. Specially, plantisolated lectins that are well characterized and used as new bioreceptors play a pivotal role in many biosensing devices due to their exquisite specificity for their cognate carbohydrates on the bacterial cell surfaces [19]. Furthermore, the molecular size of lectins is much smaller than antibodies; thus, they allow higher densities of carbohydrate-sensing elements leading to higher sensitivity and lower nonspecific adsorption [20]. Therefore, in our present investigation, we screened different plant lectins for the rapid detection L. monocytogenes in dairy products.

\section{Materials and Methods}

\section{Procurement and maintenance of culture}

Different strains of Listeria monocytogenes 15313 (serotype 4b), 13932 (serotype 4b), 19111 (serotype 1/2a), 19115 (serotype 4b), 19118 (4e) and BAA 751 [American type culture collection (ATCC)], Manassas, Virginia), L. ivanovii 19119 (ATCC), Listeria innocua 33090 (ATCC), Enterococcus faecalis 14506 (ATCC), Staphylococcus aureus (ATCC), Bacillus cereus 14569 (ATCC), Lactobacillus GG [National collection of Dairy cultures (NCDC)], E. coli 25992 (ATCC), Salmonella choleraesuis 10708 (ATCC), Enterobacter aerogenes 13048 (ATCC), Klebsiella pneumonia 138 (NCDC), Citrobacter freudenii ATCC 43864, Yersinia enterocolitica ATCC 23715 and Serratia marcescens 13880 (ATCC) used in this study were procured from their respective mentioned sources and checked for purity. Cultures were activated by incubating at $37^{\circ} \mathrm{C}$ for overnight followed by streaking on respectively; selective agars reported in Table 1, which were procured from Himedia Chemicals, Mumbai. Incubations were done overnight at $370 \mathrm{C}$ in a shaker incubator (New Brunswick ${ }^{\mathrm{TM}}$ Innova ${ }^{\circledR}$ 42/42R, Eppendorf, Germany). A single pure colony from a respective selective medium after microscopic examination was done to check the purity of the culture was picked up and maintained on its respective maintenance media by routine sub-culturing after every 15 days. Pure colonies from a selective medium of each test organism was transferred to $5.0 \mathrm{ml}$ of respective maintenance broth (Table 1) and incubated for $24 \mathrm{hrs}$ at $37^{\circ} \mathrm{C}$. The culture broths were then centrifuged at $8994 \times \mathrm{g}$ (5810R, Eppendorf, Germany) for $5 \mathrm{~min}$ at $10^{\circ} \mathrm{C}$. The cell pellet was washed once with $10 \mathrm{mM}$ phosphate buffer (PB), pH 6.8 in $2.0 \mathrm{~mL}$ eppendorf tubes to eliminate broth components. Final suspension for each test organism was prepared in $10 \mathrm{mM} \mathrm{PB}, \mathrm{pH} 6.8$ and the concentration of each organism was set to an OD of 1.0 using Tecan M pro 200 multimode plate reader (Bioscreen Instruments, Chennai, India) for further agglutination assays [21,22].

Table 1: Selective and maintenance medium used for L. monocytogenes and other contaminants [need to spell out the acronyms used for maintenance broth, selective medium, and maintenance media].

\begin{tabular}{|c|c|c|c|c|c|}
\hline Cultures & Strains & $\begin{array}{l}\text { Procured } \\
\text { from }\end{array}$ & Maintenance Broth & Selective Media & $\begin{array}{l}\text { Maintenance } \\
\text { Media }\end{array}$ \\
\hline Listeria & $\begin{array}{c}\text { L. monocytogenes 15313, } \\
\text { 13932, 19111, 19115, 19118, } \\
\text { BAA 751; L. ivanovii 1911, L. } \\
\text { innocua } 33090\end{array}$ & ATCC & Brain Heart Infusion & $\begin{array}{l}\text { Polymyxin-Acriflavin-Lithium } \\
\text { chloride-Ceftazidime-Aesculin- } \\
\text { Mannitol (PALCAM) agar }\end{array}$ & $\begin{array}{c}\text { Brain Heart } \\
\text { Infusion agar (BHI) } \\
\text { slants }\end{array}$ \\
\hline Enterococci & E. faecalis 14506 & ATCC & & Citrate azide agar & BHI slants \\
\hline Lactobacilli & Lactobacillus GG 347 & NCDC & $\begin{array}{l}\text { deMann Rogosa } \\
\text { Sharpe (MRS) }\end{array}$ & MRS agar & MRS agar slants \\
\hline Bacillus & Bacillus cereus 11778 & ATCC & Nutrient broth & Bacillus cereus Agar & $\begin{array}{l}\text { Nutrient agar (NA) } \\
\text { slants }\end{array}$ \\
\hline Staphylococcus & S. aureus 9144 & ATCC & Brain Heart Infusion & Baird Parker Agar & BHI agar slants \\
\hline Escherichia & E. coli 25922 & ATCC & \multirow{7}{*}{ Nutrient broth } & Violet red bile agar (VRBA) & NA slants \\
\hline Salmonella & S. choleraesuis 10708 & ATCC & & Xylose lysine deoxycholate agar & NA slants \\
\hline Enterobacter & E. aerogenes 13048 & ATCC & & VRBA & NA slants \\
\hline Klebsiella & K. pneumoniae 138 & NCDC & & VRBA & NA slants \\
\hline Citrobacter & Citrobacter freundii & ATCC & & VRBA & NA slants \\
\hline Yersinia & Y. enterocolitica 23715 & ATCC & & VRBA & NA slants \\
\hline Serretia & S. marcescens 14756 & ATCC & & VRBA & NA slants \\
\hline
\end{tabular}


Screening of lectin for the specific binding with $L$. monocytogenes

Ten lyophilized lectins $(1 \mathrm{mg} / \mathrm{mL})$ such as Canavalia ensiformis (Con A), wheat germ agglutinin (WGA), Helix pomatia agglutinin (HPA), Pisium sativum (PSL), Arachis hypogaea (PNA), Ulex europaeus (UEL), Lens culinaris (LCL), Sambucus nigra (SNL), Maackia amurensis (MAL) and Phytolaccka Americana (PAL) were procured from Sigma Aldrich Chemicals Pvt Ltd., (Bengaluru, India) and dissolved individually in $\mathrm{PB}(10 \mathrm{mM}, \mathrm{pH} 6.8)$ followed by dilution to an optimum concentration of $200 \mu \mathrm{g} / \mathrm{mL}$. About $50 \mu \mathrm{L}$ of each lectin mixed with $50 \mu \mathrm{L}$ of L. monocytogenes cells at a concentration of 107 CFU per ml [(15313 (4b), 13932 (4b), 19111 (1/2a), 19115 (4b), 19118 (4e) \& BAA 751, RM-17 and RM-26)] in suspension were added together in $\mathrm{U}$ bottom microliter plate procured from Cole-Parmer (Mumbai, India). For a control, $L$. monocytogenes suspension was mixed with $50 \mu \mathrm{l}$ of PB in separate wells of $U$ Bottom microliter plate. All the experiment related to screening of lectin was done in triplicate $(n-=3)$. The microtiter plate was incubated at $37^{\circ} \mathrm{C}$ for $10 \mathrm{~min}$ in a shaker incubator (New Brunswick ${ }^{\mathrm{TM}}$ Innova ${ }^{\circledR}$ 42/42R, Eppendorf, Germany). After incubation, the microtitre plate was observed for agglutination visually [21], with the appearance of ground glass in transmitted light was taken as a negative reaction. Further, agglutinated cells were stained with crystal violet dye (Himedia, Mumbai, India) and observed under iXon Electron Multiplying charged coupled device (EMCCD) using light microscopy (ANDOR, Bengaluru). Images of agglutination were captured using a camera Sony Lightshot (Sony New Delhi, India). The microscopic observation taken during the agglutination assay was interpreted as per Naughton et al. [22], who described $80-90 \%$ agglutination as $+4,60-70 \%$ agglutination as $+3,40-50 \%$ as $+2,10-20 \%$ as +1 and $<10 \%$ as negative.

\section{Screening of lectin with Gram positive and Gram negative bacteria}

Further, all ten lectins were screened individually with different Gram positive bacteria (GPB) other than L. monocytogenes (Listeria ivanovii, Listeria innocua, Bacillus cereus, Staphylococcus aureus, Lactococcus lactis, Lactobacillus fermentum, Leuconostoc mesenteroides) and Gram negative bacteria (GNB) [Escherichia coli 25992 (ATCC), Salmonella choleraesuis 10708 (ATCC), Enterobacter aerogenes 13048 (NCDC), Klebsiella pneumonia 138 (NCDC), Citrobacter freudenii ATCC 43864, Yersinia enterocolitica ATCC 23715 and Serratia marcescens 13880 (ATCC)] in respective growth medium for agglutination activity $[21,22]$.

\section{Scanning electron microscopic study of agglutination}

The selected WGA lectin was further studied for agglutination with L. monocytogenes using scanning electron microscopy (SEM)) using the method described by Facinelli et al. [21]. After

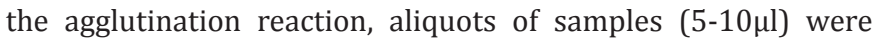
air dried on glass cover slips and fixed in a solution of $2.5 \%$ glutaraldehyde in $75 \mathrm{mM}$ phosphate buffer, ( $\mathrm{pH} 7.4$ ) for 45-60 min. The fixed specimen was rinsed three times each for $15 \mathrm{~min}$, in 75 $\mathrm{mM}$ phosphate buffer ( $\mathrm{pH}$ 7.4). After the rinsing, samples were serially dehydrated in ethanol concentrations of 30, 50, 70, 80, 90 and $100 \%$ each for $15 \mathrm{~min}$, respectively. After this step, the sample was air dried, and the specimen sample was mounted on a stub followed by gold coating. In gold coating, samples were encrusted with gold at approximately 100-200 $\AA$ thickness using an ion coater (Hitachi IB-3, Tokyo, Japan). The ion current was kept at $6-8 \mathrm{~mA}$ with a fine vacuum of $0.05-0.07$ torr for 2-4 minutes. The coated samples were then visualized using a SEM at 700030,000 X magnifications (EVO® 18, Carl ZEISS Special Edition, Cambridge,-UK).

\section{Effect of sugars on lectin banding activity of $L$. monocytogenes}

Different sugars such as D-glucose (Glc), D-galactose (Gal), D-mannose (Man) and N-acetylglucosamine (NAGA) were screened for sugar specificity of selected lectins with $\mathrm{L}$. monocytogenes, using an inhibition assay [21,22]. Different concentrations of sugar $(0.1$ to $1 \mathrm{M}, 50 \mu \mathrm{l})$ were mixed with $50 \mu \mathrm{l}$ of each selected lectin followed by incubation for $1 \mathrm{hr}$ at $37^{\circ} \mathrm{C}$. After incubation, the mixture was studied for agglutination using L. monocytogenes [21,22]. Further, the percentage inhibition of agglutination activity was calculated by the ratio of agglutination activity lectin in the presence of the sugar to the agglutination observed with L. monocytogenes without addition of sugar.

\section{Results and Discussion}

\section{Screening of lectin for the specific binding with $L$. monocytogenes}

Among ten lectins screened for agglutination activity with different strains of L. monocytogenes cited above, HPA and WGA showed $80-90 \%$ activity with $L$. monocytogenes cells, having 8.38 log counts per $\mathrm{mL}$, followed by $60-70 \%$ activity with AHL, $40-50 \%$ activity with UEL, LCL and SNA; $10-20 \%$ activity with PAL and MAL, respectively as shown in Figure 1 . However, $<10 \%$ agglutination was observed with PSA. Figure 2 depicts the microscopic observation of agglutination of L. monocytogenes with different lectins by simple staining technique and analysis through EMCCD. Maximum agglutination with all strains of $L$. monocytogenes was observed with WGA followed by HPA, AHA, UEL, MAA, LCL, PAL, SNA, CON A and PSA with an HA of $84.4 \%, 70 \%, 51.25 \%$, $39.375 \%, 31.25 \%, 30.625 \%, 26.25 \%, 18.75 \%, 16.25 \%$ and $9.375 \%$, respectively. The maximum HA was observed with WGA in the presence of different strains of L. monocytogenes; therefore, WGA was selected for further screening study with Gram positive and Gram negative bacteria. L. monocytogenes could interact with HPA and WGA whereas, Arachis hypogaea (PNA), Glycine max (SBA), and Vicia villosa (VVA) lectins failed to agglutinate L. monocytogenes 15313 or any of the other test strains under the experimental conditions [21]. Another study conducted by Facinelli et al. [23] observed that 26 strains of L. monocytogenes 15313 were agglutinated by WGA, 17 by Griffonia simplicifolia-1 (GS-1) by RCA-I (ricinus communis agglutinin), and nine by HPA. Therefore, WGA from TVA was selected for further study.

\section{Screening of Gram positive and Gram negative bacteria for Lectin agglutination}

All ten lectins including WGA were also further checked for agglutination activity with GPB (other strains of Listeria spp. such as Listeria innocua 33090, Listeria ivanovii 19119, Enterococcus 
faecalis, Bacillus cereus and Staphylococcus aureus)and GNB (E. coli 0157:H7 Escherichia coli 25992 (ATCC), Salmonella choleraesuis 10708 (ATCC), Enterobacter aerogenes 13048 (NCDC), Klebsiella pneumonia 138 (NCDC), Citrobacter freudenii ATCC 43864, Yersinia enterocolitica ATCC 23715 and Serratia marcescens 13880 ). In our study, no or $<10 \%$ HA activity was observed with WGA among GPB as shown in Figure 3. Figure 4 depicts the WGA agglutination activity in GNB wherein, zero agglutination was observed among E. coli, E. coli 0157:H7, Enterobacter, and Serretia whereas, in the case of Salmonella and Klebsiella HA was 10-20\% followed by $45 \%$ activity in Citrobacter. Jebor et al. [24] found HA of PSA immobilized with glutaraldehyde in presence of Bacillus, Salmonella, Staphylococcus, Streptococcus and Pseudomonas as compared to E. coli, Enterobacter on the basis of increase in optical density at $660 \mathrm{~nm}$ and through microscopic examination using Gran staining technique. In an another study by Payne et al. [25] wherein they immobilized Helix pometia, Canavalia ensiformis, Agaricus bisporus and Triticum vulgaris i.e., WGA on magnetic microspheres for the evaluation of binding activity with L. monocytogenes, S. aureus, Salmonella and E. coli and observed $87-100 \%$ agglutination activity with $L$. monocytogenes in the presence of WGA, $>92 \%$ binding with $S$. aureus in the presence of HPA, but poor binding with GNB in the presence of WGA and HPA. The membrane of GNB has not shown increased reactivity to WGA when exposed to increased salinity of the reaction mixture due to loosening of the rigid structure and it has improved the staining with the lectin [26]. Based on these results, it was confirmed that the WGA was highly selective for L. monocytogenes. Therefore, WGA was selected for further study.

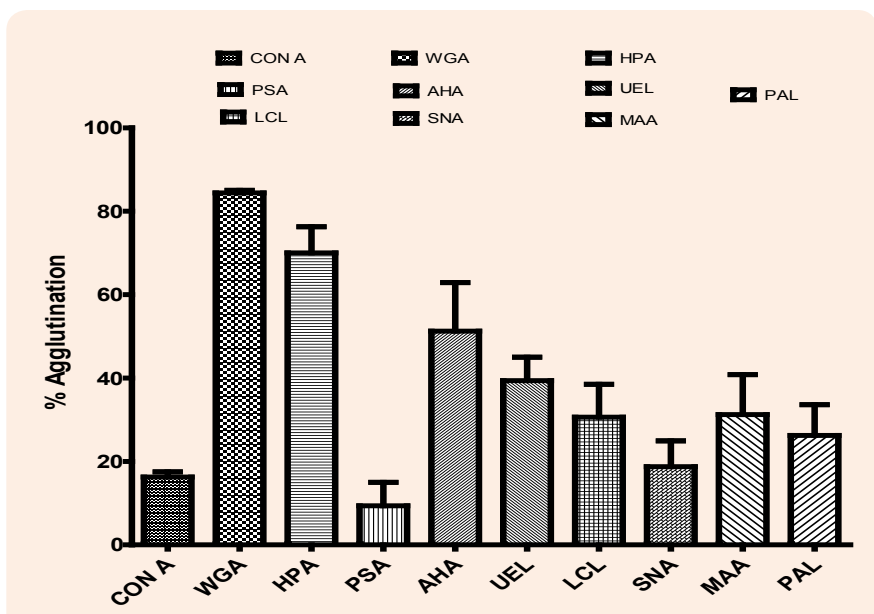

Figure 1: Agglutination activity of different strains of L. monocytogenes with various lectins.

\section{Scanning electron microscopic study of agglutination}

Figure $5 \mathrm{a} \& \mathrm{~b}$ shows the arrangements of the L. monocytogenes ATCC 15313 in the presence (Figure 5a) or absence of (Figure 5b) $200 \mu \mathrm{g} / \mathrm{ml}$ of WGA lectin. Figure 5c shows the pure WGA lectin without L. monocytogenes ATCC 15313 cells. The electron microscopic observations depict that the control cells of L. monocytogenes ATCC 15313 cells were not induced to aggregate but they arranged in singles and pairs. However, the $L$. monocytogenes ATCC 15313 cell aggregation in the presence of lectin appeared to be gapped and patchier, but, cell rupture was only observed in some cases (as shown in Figure 5).

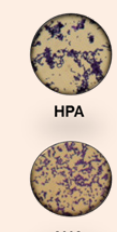

AHA

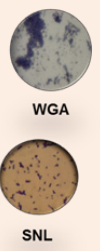

SNL

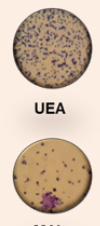

MAL

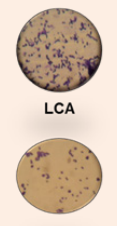

PAL

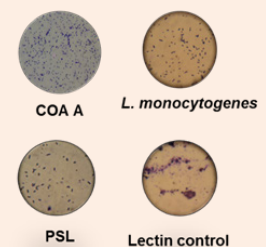

Lectin control
Figure 2: Light microscopic observation of agglutination of $L$. monocytogenes 15313 with different lectins by crystal violet staining. Lectin control contains $200 \mu \mathrm{g} / \mathrm{ml}$ lectin dissolved in $10 \mathrm{mM}$ phosphate buffer, pH 6.8 .

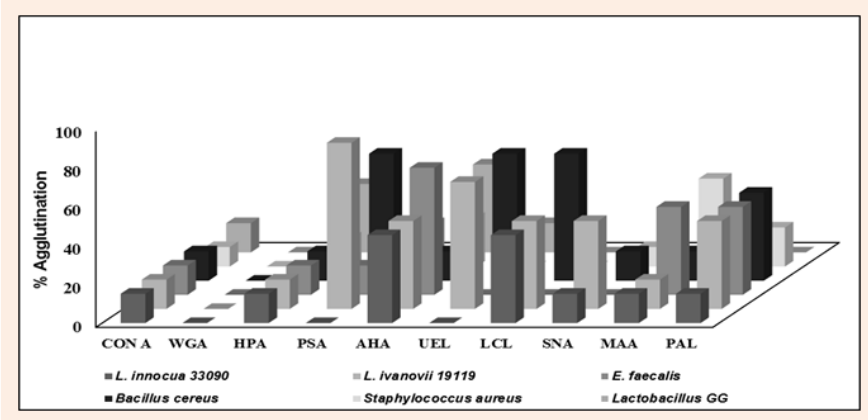

Figure 3: Lectin agglutination activity of different Gram positive bacteria.

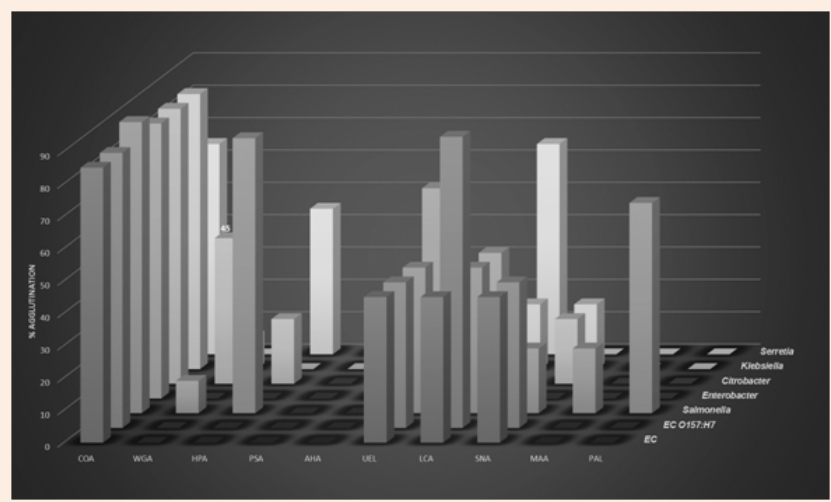

Figure 4: Lectin agglutination activity of different Gram negative bacteria.

\section{Inhibition of lectin binding activity of L. monocytogenes by different sugars}

Carbohydrate inhibition studies were performed with L. monocytogenes 15313 using Glc, Gal, Man and NAGA. The WGA was consistently inhibited in presence of NAGA with an inhibition $\%$ of $0,35,50,80$ and $100 \%$ inhibition of WGA binding activity 
at $0.1,0.25,0.5,0.75$ and $1 \mathrm{M}$ concentration, respectively. In case of glucose, the \% inhibition was $0,25,35,55$ and 100 and was observed at $0.1,0.25,0.5,0.75$ and $1 \mathrm{M}$ concentration, respectively. Whereas, in the case of galactose, the inhibition was only observed at 0.75 and $1 \mathrm{M}$ concentration with 75 and $90 \%$ inhibition respectively, the least inhibition was observed with mannose where only $25 \%$ inhibition was found at $1 \mathrm{M}$ concentration (Figure 6). The maximum or complete inhibition was observed in presence of NAGA, and indicates that the binding of L. monocytogenes cells to WGA lectin was highly specific for NAGA followed by glucose, galactose and mannose. The binding of L. monocytogenes cells to WGA was partially inhibited by glu and gal, suggesting that glu and gal moieties are also present on the cell surface structure of L. monocytogenes. The mannose inhibition of WGA agglutination was supported by Neu et al. [27]

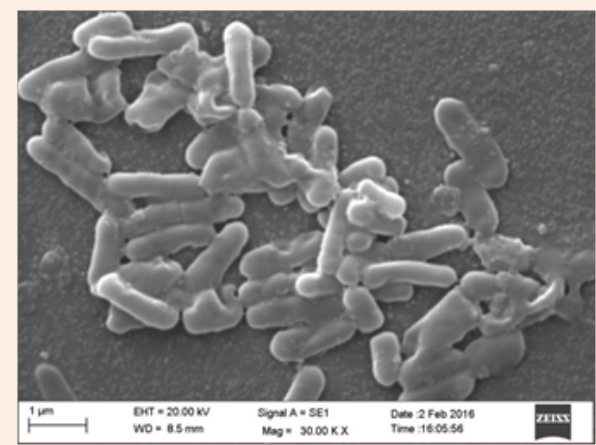

Figure 5a: SEM image of lectin agglutination with $L$. monocytogenes ATCC 15313.

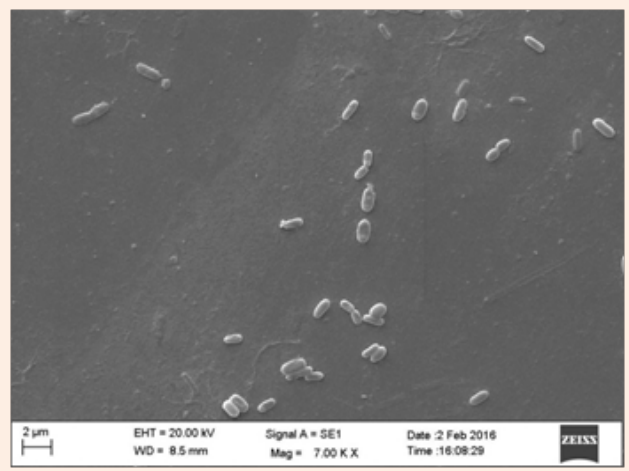

Figure 5b: SEM image of L. monocytogenes ATCC 15313 in the absence of lectin.

\section{Conclusion}

In our present study, L. monocytogenes has shown strong agglutination activity (HA) with WGA followed by HPA, AHA, UEL, MAA, LCL, PAL, SNA, CON A and PSA. The GPB have also shown HA with all lectins screened except WGA wherein HA was $<10 \%$. The GNB, especially Enterobacteriaceae groups, haven't shown HA in presence of WGA except Citrobacter (45\%), Klebsiella and Salmonella (10-20\%) and maximum HA was observed in presence that wide range of carbohydrates such as glu, gal, man, arabinose, rhamnose, etc. can inhibit the WGA binding in biofilm and about $31 \%$ inhibition in WGA binding of biofilm were seen under florescent microscopes using FITC-labelled WGA staining of biofilm. This work is supporting our claim of mannose inhibition of WGA binding with L. monocytogenes. Volf et al. [28] \& McGreal et al. [29] reported that carbohydrate binding specificity is an important factor in the recognition of carbohydrates on pathogen cell surfaces. Further, Loessner et al. [30] has shown that WGA lectin requires terminal NAGA residues present on the teichoic acid of the bacterial wall (WTAs) for attachment. Nir Paz et al. [31] described the binding of fluorescently labeled WGA, a lectin that specifically binds terminal NAGA residues in cell wall polymers using Alexa Fluor 594®. They found that WGA was able to bind wild strains of L. monocytogenes such as 10403S and DP-L5415.

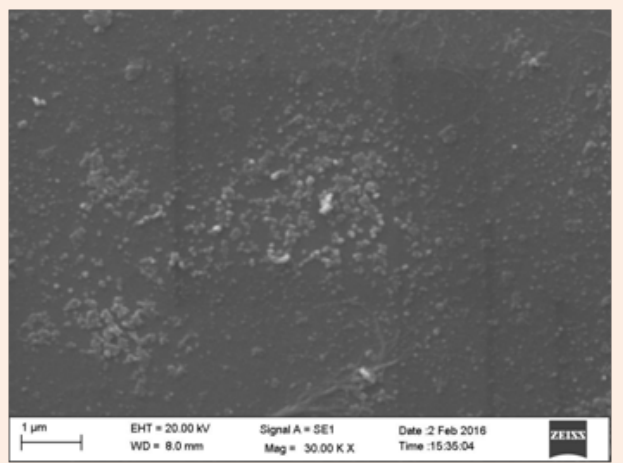

Figure 5c: SEM image of WGA lectin.

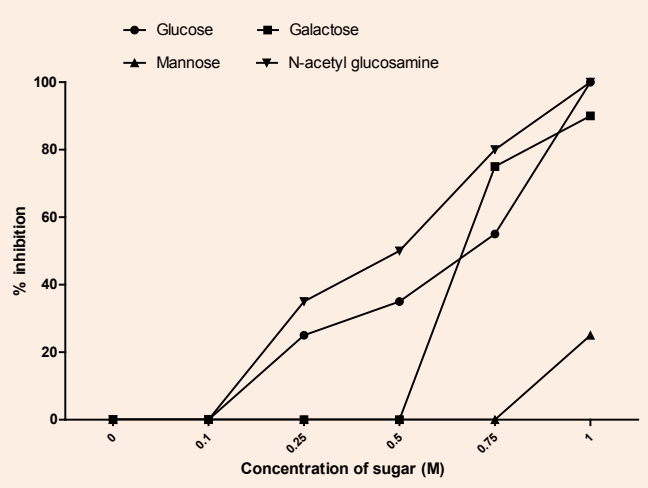

Figure 6: Inhibition of agglutination of $L$. monocytogenes by different sugars.

of ConA. The WGA activity with L. monocytogenes was also well established with SEM and by sugar inhibition assays. Based on the inhibition test, it was clear that WGA was specifically bound with NAGA followed by Glc, Gal was present on the cell wall of L. monocytogenes and whereas in case of man it was only $25 \%$ inhibition. From the above results, it is clear that WGA may be used as a marker protein for the detection of L. monocytogenes in milk and milk products. 


\section{Acknowledgment}

This work was supported by the National Agriculture Innovation Project (NAIP), Niche area of Excellence (NAE), Outreach Programme (ICAR). The authors express their appreciation to personnel to Dr. S. K. Tomar, Principal Scientist \& Incharge, SEM lab and Dr. Vaibao Lule Patil, PhD scholar, Dairy Microbiology Division for SEM study of lectin agglutination with L. monocytogenes. Director and Vice Chancellor, ICAR-National Dairy Research Institute (NDRI), Deemed University, Karnal for providing necessary research facility for conducting research work on Lectin binding of L. monocytogenes.

\section{References}

1. Jalali M, Abedi D (2008) Prevalence of Listeria Species in Food Products in Isfahan, Iran. Int J Food Microbiol 122 (3): 336-340.

2. Schlech WF, David A (2000) Foodborne Listeriosis. Clin Infect Dis 31(3): 770-775.

3. Walker SJ, Archer P, Banks JG (1990) Growth of L. monocytogenes at refrigeration temperatures. J Appl Bacteriol 68(2): 157-162.

4. Wong ACL (1998) Biofilms in food processing environments. J Dairy Sci 81: 2765-2770.

5. Ragimbeau DG, Malle P (2001) Use of PFGE typing for tracing contamination with $L$. monocytogenes in three cold smoked salmon processing plants. Int J Food Microbiol 64(1-2): 51-61.

6. Ryser ET, Marth EH (1999) Listeria, Listeriosis and Food Safety. (2 ${ }^{\text {nd }}$ edn), Marcel Dekker Inc New, USA.

7. Bula CJ, Bille J, Glauser MP (1995) An epidemic of food-borne listeriosis in western Switzerland: description of 57 cases involving adults. Clin Infect Dis 20: 66-72.

8. Gaya P, Sanchez J, Medina M, Nunez M (1998) Incidence of $L$. monocytogenes and other Listeria species in raw milk produced in Spain. Food Microbiol 15: 551-555.

9. Filiousis G, Anders J, Joachim F, Vincent P (2009) Prevalence, genetic diversity and antimicrobial susceptibility of $L$. monocytogenes isolated from open-air food markets in Greece. Food control 20: 314317.

10. CDC (2013) Multistate Outbreak of Listeriosis Linked to Crave Brothers Farmstead Cheeses (Final Update). Centre for diseases control and prevention, USA.

11. Rudolf M, Scherer S (2001) High incidence of L. monocytogenes in European red smear cheese. Int J Food Microbiol 63(1-2): 91-99.

12. Colak H, Hamparsun H, Enver BB, Beyza U (2007) Prevalence of $L$. monocytogenes and Salmonella spp. in Tulum cheese. Food control 18 (5): 576-579.

13. CDC (2015) Multistate Outbreak of Listeriosis Linked to Raw Milk Produced by Miller's Organic Farm in Pennsylvania. Centre for diseases control and prevention, USA.

14. Meloni D, Pietro G, Anna M, Francesca P, Mansel G, Rina M (2009) Listeria Monocytogenes in RTE Foods Marketed in Italy: Prevalence and Automated Ecori Ribotyping of the Isolates. Int J Food Microbiol 129(2): 166-173.
15. WHO (2002) Emerging Food-borne Diseases. World Health Organization, Switzerland, 124: 1-3.

16. Paul C, Li Y, Cui T, Ruan R, Ruan R (2013) Nanoparticles based sensors for rapid detection of foodborne pathogens. Int J Agri Biolog Eng 6(1)-28-35.

17. Goldstein IJ, Hayes CE (1978) The lectins: carbohydrate-binding proteins of plants and animals. Adv Carbohydr Chem Biochem 35: 127-340.

18. Ambrosi M, Cameron NR, Davis BG (2005) Lectins: tools for the molecular understanding of the glycocode. Org Biomol Chem 3(9): 1593-1608.

19. Rahaie M, Kazemi SS (2010) Lectin-Based Biosensors: As Powerful Tools in Bioanalytical Applications. Biotechnol 9(4): 428-443.

20. Wang Y, Zunzhong Y, Yibin Y (2012) New trends in impedimetric biosensors for the detection of foodborne pathogenic bacteria. Sensors 12(3): 3449-3471.

21. Facinelli B, Eleonora G, Chiara C, Pietro EV (1994) Interactions with lectins and agglutination profiles of clinical, food, and environmental isolates of Listeria. J Clin Microbiol 32(12): 2929-2935.

22. Naughton PJ, Grant G, Bardocz S, Pusztai A (2000) Modulation of Salmonella infection by the lectins of Canavalia ensiformis (Con A) and Galanthus nivalis (GNA) in a rat model in vivo. J Appl Microbiol 88(4): 720-727.

23. Facinelli B, Giovanetti E, Magi G, Biavasco F, Varaldo PE (1998) Lectin reactivity and virulence among strains of L. monocytogenes determined in vitro using the enterocyte-like cell line Caco-2. Microbiology 144(1): 109-118.

24. Jebor MA, Yasser HJ (2013) Interaction of Some Pathogenic Bacteria with Phaseolus Vulgaris L. Seeds Lectin (as Biosensor). Int J Biosci Bioche Bioinforma 3(3): 239-241.

25. Payne MJ, Shona C, Patchett RA, Kroll RG (1992) The Use of Immobilized Lectins in the Separation of S. aureus, E. coli, Listeria and Salmonella Spp. From Pure Cultures and Foods. J Appl Bacteriol 73(1): 41-52.

26. Jespersen L, Holm C (2002) Method for Differential Analysis of Bacteria in a Sample. Google Patents W02002057482 A2.

27. Neu TR, Swerhone GD, Lawrence JR (2001) Assessment of lectinbinding analysis for in situ detection of glycoconjugates in biofilm systems. Microbiol 147(2): 299-313.

28. Volf P, Skarupova S, Man P (2002) Characterization of the lectin from females of Phlebotomus duboscqi sand flies. Eur J Biochem 269(24): 6294-6301.

29. McGreal EP, Martinez Pomares L, Gordon S (2004) Divergent roles for C-type lectins expressed by cells of the innate immune system. Mol Immunol 41(11): 1109-1121.

30. Loessner MJ, Kramer K, Ebel F, Scherer S (2002) C-terminal domains of L. monocytogenes bacteriophage murein hydrolases determine specific recognition and high-affinity binding to bacterial cell wall carbohydrates. Mol Microbiol 44(2): 335-349.

31. Nir Paz R, Eugster MR, Zeiman E, Loessner MJ, Calendar R (2012) L. monocytogenes tyrosine phosphatases affect wall teichoic acid composition and phage resistance. FEMS Microbiol Let 326(2): 151160. 